\title{
SELEÇÃO DE JULGADORES E AVALIAÇÃO DE DIFERENÇA SENSORIAL ENTRE EXTRATOS HIDROSSOLÚVEIS DA AMÊNDOA DA CASTANHA DE CAJU (Anacardium occidentale L.)
}

\author{
ANACRISTINA DA SILVAMORAIS* \\ MARIA DO CARMO PASSOS RODRIGUES** \\ MARINA CABRAL REBOUÇAS*** \\ MARIAFLÁVIAAZEVEDO DA PENHA****
}

\begin{abstract}
Aplicou-se o teste triangular em 12 julgadores selecionados para verificar diferença significativa $(p \leq 0,001)$ na utilização de amêndoas da castanha de caju quebradas (classificadas como bandas, batoques e pedaços) do tipo 1, como matéria-prima para obtenção de extrato hidrossolúvel. As amostras do extrato hidrossolúvel foram produzidas triturando-se amêndoas da castanha de caju com diferentes proporções de água mineral. Na etapa de seleção dos julgadores, mediante teste triangular, as proporções amêndoas:água foram de $1: 6,1: 8$ e $1: 10$ e para o teste discriminativo de 1:8, havendo diferença entre as amostras somente em relação à classificação das amêndoas da castanha de caju. As amostras foram envasadas em embalagens de polietileno, pasteurizadas e armazenadas sob refrigeração. Os resultados foram estatisticamente avaliados pela análise sequencial de Wald, segundo o método gráfico. A decisão baseou-se no teste de hipóteses: $H_{0}$ : $p 1 \leq p 0 ; H_{1}: p 1>p 0$, sendo utilizados os valores $\mathrm{p} 0=0,33, \mathrm{p} 1=0,66$ e para os riscos $\alpha=0,05$ e $\beta=0,05$. Na avaliação de diferença significativa $(p \leq 0,001)$ pelo teste triangular, as amêndoas classificadas como bandas e batoques do tipo 1 não apresentaram diferença, mas diferiram das amêndoas classificadas como pedaços do tipo 1. Considerando que as amêndoas classificadas como pedaços apresentam menor percentual de integridade que as demais (estando mais suscetíveis à oxidação da fração lipídica) indica-se a utilização de bandas e batoques como matéria-prima para a elaboração de extrato hidrossolúvel.
\end{abstract}

PALAVRAS-CHAVE: ANÁLISE SEQUENCIAL; TESTE TRIANGULAR; EXTRATO HIDROSSOLÚVEL; AMÊNDOA DA CASTANHA DE CAJU.

* $\quad$ Mestre em Ciência e Tecnologia de Alimentos, Professora, Curso de Tecnologia em Alimentos, Instituto Federal de Educação, Ciência e Tecnologia do Ceará (IFCE), Limoeiro do Norte, CE (e-mail: anacmorais@ifce.edu.br).

** Doutora em Tecnologia de Alimentos, Professora, Departamento de Tecnologia de Alimentos, (UFC), Fortaleza, CE (e-mail: carminha@ufc.br).

*** Mestranda em Ciência e Tecnologia de Alimentos, ex-bolsista PIBIC/CNPq, UFC, Fortaleza, CE (e-mail: marina_reboucas@hotmail.com).

*** Graduanda em Engenharia de Alimentos, bolsista PIBIC/CNPq, UFC, Fortaleza, CE (e-mail: mflaviaaz@gmail.com). 


\section{INTRODUÇÃO}

A castanha, o verdadeiro fruto do caju, é a sua parte mais importante em termos comerciais. Dela aproveitam-se, principalmente, a amêndoa (ACC) e o líquido da casca da castanha (LCC) (PARREIRAS, 2007).

O cultivo e processamento da castanha de caju são realizados predominantemente no estado do Ceará, principal exportador brasileiro da amêndoa da castanha de caju (NASSAR, MARINO e HERRMANN, 2001; FIGUEIRÊDO JÚNIOR, 2006). No entanto, o processo de beneficiamento utilizado resulta em maior percentual de amêndoas quebradas se comparado aos demais processos existentes. Tal fato se reflete na depreciação do preço das amêndoas quebradas em relação às inteiras (PINHEIRO et al., 2006; ANDRADE NETO, 2006).

A aferição da qualidade da castanha de caju in natura e da sua amêndoa é regida pela Portaria n 51, de 06 de março de 2009, que visa estabelecer o regulamento técnico da amêndoa da castanha de caju, definindo o seu padrão oficial de classificação, com os requisitos de identidade e qualidade, a amostragem, o modo de apresentação e a marcação ou rotulagem (BRASIL, 2009).

Nas exportações da amêndoa da castanha de caju, os fatores-chave de compra não variam significativamente de um país para o outro e são estabelecidos, principalmente, pelos importadores. A qualidade das amêndoas em relação às condições contratuais de integridade, tamanho, cor e sabor obedecem aos padrões de tolerância definidos pela Association of Food Industries (AFI) visando a exportação (USAID, 2006).

De acordo com a AFI (1999), após o beneficiamento, as amêndoas são classificadas basicamente pelo tamanho, integridade e cor. Segundo o tamanho e a integridade, as amêndoas (relação da quantidade em 453,59 g, equivalente a uma libra) podem ser classificadas, em ordem decrescente, como: Inteiras (Wholes-W), Bandas (Splits-S), Batoques (Butts-B), Pedaços (Pieces-P), Grânulos (Granules-G), Xerém (X) e Farinha (F).

A amêndoa da castanha de caju será classificada em tipos, definidos em função da sua cor e dos limites máximos de tolerância de defeitos previstos na Instrução Normativa, constante da Portaria 51 . 0 Tipo 1 (primeira qualidade) envolve amêndoas de cor alva ou marfim pálido; o Tipo 2 (segunda qualidade) amêndoas de cor marfim fechada ou ligeiramente amarelada; o Tipo 3 (terceira qualidade) inclui amêndoas de cor creme ou ligeiramente tostada, podendo ser ainda ligeiramente arroxeada e o Tipo 4 (quarta qualidade) é constituído de amêndoas com cor idêntica à dos tipos 1 e 2, admitindo-se pontos pretos (brocadas) ou pequenas manchas em um ou em ambos os cotilédones (BRASIL, 2009; AFI, 1999).

Segundo o United States Department of Agriculture (USDA, 2008), a amêndoa da castanha de caju crua apresenta $18,22 \%$ de proteína, $43,85 \%$ de lipídios totais, 5,20\% de umidade, $2,54 \%$ de cinzas, $30,19 \%$ de carboidratos e $23,49 \%$ de amido, sendo muito rica em minerais e vitaminas essenciais.

Lima, García e Lima (2004); Lameira, Coelho e Mothe (1997) e Souza Filho et al. (1991), estudando a fração lipídica da amêndoa da castanha de caju, verificaram a predominância de ácidos graxos insaturados, indicando sua suscetibilidade à oxidação (rancificação). Tendo em vista que as amêndoas quando quebradas apresentam maior área de contato com o oxigênio atmosférico (agente da oxidação) acredita-se que haja diferença sensorial entre algumas classificações em relação ao percentual de integridade.

Testes triangulares são extensivamente utilizados para a análise de alimentos visando determinar se diferenças entre dois produtos podem ser sensorialmente detectadas. Os julgadores precisam selecionar a amostra diferente num grupo de três, sendo duas idênticas e uma diferente. A interpretação estatística do teste baseia-se na lei binomial com $p=1 / 3$ (hipótese nula, não há diferença significativa entre os dois produtos testados) (BI, 2001; ABNT, 1993). Quando o número de respostas corretas do teste triangular iguala ou excede o valor crítico compatível em dado nível de probabilidade, a hipótese nula é rejeitada e diferenças significativas entre amostras são inferidas (CARBONELL, CARBONELL e IZQUIERDO, 2007).

Os julgadores do teste triangular devem ser previamente recrutados e selecionados mediante uma 
série de testes visando familiarizá-los com os métodos e produtos, além de permitir a seleção dos candidatos com adequada acuidade sensorial (FARIA e YOTSUYANAGI, 2008). Os testes utilizados na seleção de julgadores para testes de diferença são o triangular, o pareado e o duo-trio (CHAVES, 2001).

A forma mais usual de seleção dos candidatos baseia-se na porcentagem de acertos nos testes. Aqueles que obtiverem mais de $60 \%$ de respostas corretas são selecionados. No entanto, para que esse critério seja adotado, o candidato necessita realizar entre 20 e 24 testes (FARIA e YOTSUYANAGI, 2008). A aplicação da análise sequencial de Wald na seleção apresenta como vantagem a economia de tempo e de material, tendo em vista que os candidatos com maior potencial podem ser selecionados com menor número de testes (CHAVES, 2001).

Este trabalho teve por objetivo utilizar a análise sequencial de Wald para selecionar julgadores e verificar, por meio de teste triangular se há diferença sensorial significativa (pd"0,001) na utilização de amêndoas da castanha de caju quebradas (classificadas como bandas, batoques e pedaços, do tipo 1) como matéria-prima para produção de extrato hidrossolúvel, tendo em vista a maior ou menor suscetibilidade dessas amêndoas à oxidação durante o beneficiamento.

\section{MATERIAL E MÉTODOS}

\subsection{MATÉRIA-PRIMA}

Foram utilizadas amêndoas da castanha de caju em pedaços, obtidas no comércio da cidade de Fortaleza (CE) e amêndoas doadas pela empresa Cascaju Agroindustrial S/A, classificadas como S1 (bandas tipo 1), B1 (batoques tipo 1) e P1 (pedaços tipo 1) (AFI, 1999; BRASIL, 2009).

\subsection{PREPARAÇÃO DAS AMOSTRAS}

Os extratos hidrossolúveis foram produzidos triturando-se as amêndoas da castanha de caju (ACC) com diferentes proporções de água mineral, sendo o processamento realizado conforme a Figura 1.

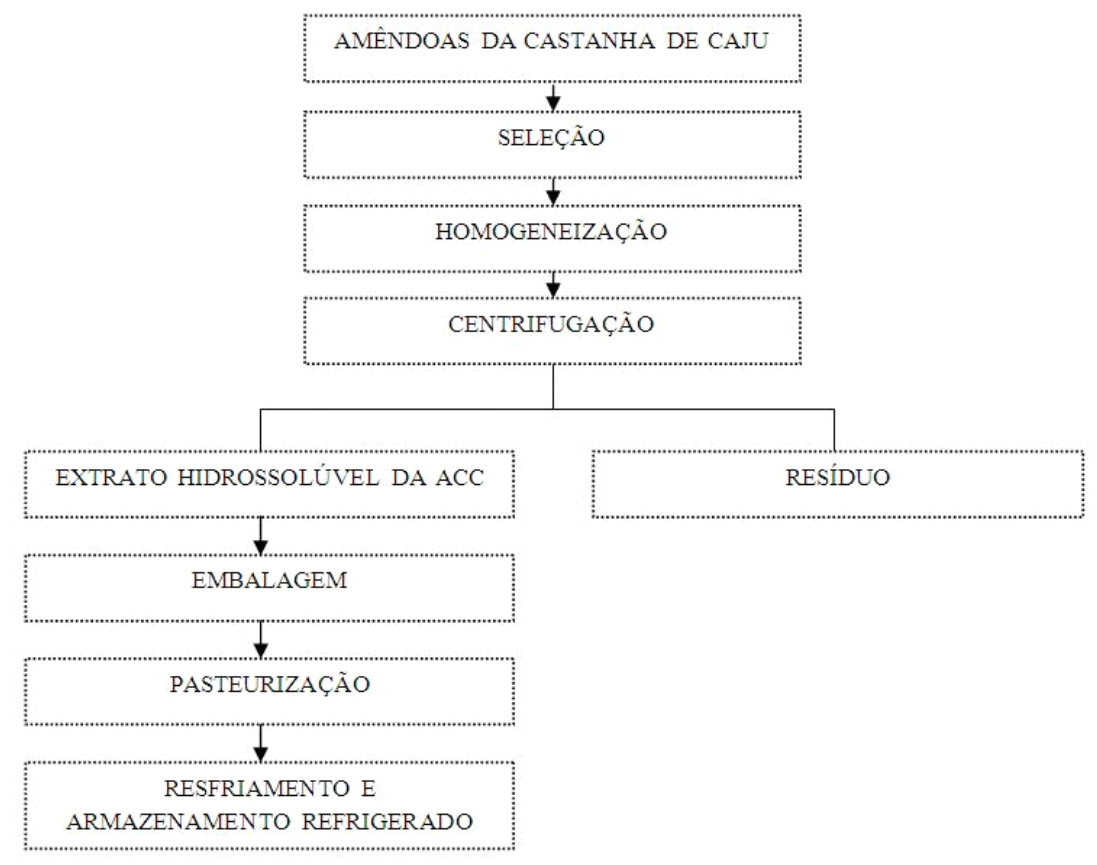

FIGURA 1 - FLUXOGRAMA DO PROCESSO DE OBTENÇÃO DO EXTRATO HIDROSSOLÚVEL DA AMÊNDOA DA CASTANHA DE CAJU (ACC) 
Os extratos foram produzidos triturando-se amêndoas da castanha de caju selecionadas com diferentes proporções de água mineral a $17000 \mathrm{rpm}$ por 3 minutos. As proporções amêndoas:água empregadas para obtenção das amostras utilizadas na etapa de seleção dos julgadores foram 1:6, 1:8 e 1:10. Para o teste discriminativo usou-se a proporção 1:8, havendo diferença entre as amostras somente em relação à classificação das amêndoas da castanha de caju.

Após a trituração, realizou-se a centrifugação por 30 segundos em baixa rotação para a remoção dos componentes mais grosseiros (resíduo) que poderiam sedimentar durante o armazenamento. As amostras foram envasadas em embalagens de polietileno com capacidade para $200 \mathrm{~mL}$, seladas termicamente. Em seguida, foram pasteurizadas a $72^{\circ} \mathrm{C} \pm 2^{\circ} \mathrm{C}$ por 20 minutos em banho-maria (FELBERG et al., 2002; CARDARELLI e OLIVEIRA, 2000), submetidas a choque térmico em banho de gelo e armazenadas sob refrigeração.

\subsection{SELEÇÃO DOS JULGADORES}

Os julgadores foram selecionados a partir de 22 voluntários, recrutados mediante aplicação de doze testes triangulares (CHAVES, 2001). Os julgadores que não foram selecionados nem rejeitados ao fim dos doze testes, realizaram testes extras. Nos seis primeiros testes utilizaram-se as amostras de extrato hidrossolúvel da amêndoa da castanha de caju preparadas nas proporções amêndoas:água 1:6 e 1:8. Nos outros seis testes, bem como nos testes extras foram utilizadas amostras preparadas nas proporções 1:6 e 1:10.

Os resultados foram estatisticamente avaliados pela análise sequencial de Wald, segundo o método gráfico. A decisão baseou-se no teste de hipóteses: $\mathrm{H}_{0}: \mathrm{p} 1 \mathrm{~d} \leq \mathrm{p} 0 ; \mathrm{H}_{1}: \mathrm{p} 1>\mathrm{p} 0$, sendo utilizados os valores $\mathrm{p} 0=0,33$ (máxima inabilidade aceitável), p1=0,66 (mínima habilidade aceitável) e para os riscos $\alpha=0,05$ (probabilidade de selecionar candidato inaceitável) e $\beta=0,05$ (probabilidade de não selecionar candidato aceitável) (CHAVES, 2001). As amostras foram apresentadas aos candidatos nas 6 possíveis combinações (AAB; BAA; ABA; BBA; BAB; $A B B)$.

\subsection{AVALIAÇÃO DE DIFERENÇA SENSORIAL ENTRE DIFERENTES EXTRATOS}

Avaliou-se mediante o teste triangular se havia diferença significativa $(\mathrm{pd} \leq 0,001)$ entre bandas (S1) e batoques (B1), bandas (S1) e pedaços (P1), batoques (B1) e pedaços (P1) (AFI, 1999) como matéria-prima para produção do extrato hidrossolúvel da amêndoa da castanha de caju. Os 12 julgadores selecionados avaliaram as três combinações de amostras de extrato hidrossolúvel em triplicata, totalizando 36 respostas para cada combinação de amostras (CHAVES, 2001). Cada julgador recebeu as amostras em ordem balanceada e casualizada (STONE e SIDEL, 1993).

\section{RESULTADOS E DISCUSSÃO}

\subsection{SELEÇÃO DE JULGADORES}

As Figuras 2 e 3 apresentam os resultados da seleção dos julgadores realizada pela análise sequencial de Wald, mediante teste triangular. As retas de aceitação e de rejeição obtidas para a seleção dos julgadores foram d1 $=2,15+0,50 \mathrm{n}$ e d0 $=-2,144+0,494 \mathrm{n}$. Dos 22 candidatos avaliados com o objetivo de compor a equipe, apenas 12 foram selecionados mediante a metodologia proposta. 


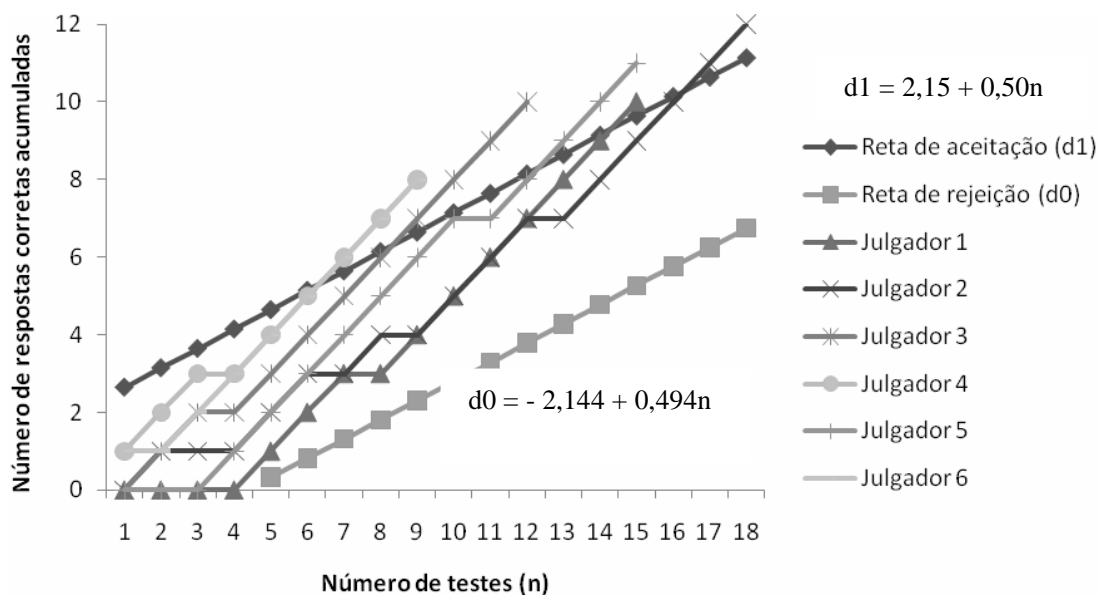

\section{FIGURA 2 - RESULTADO DO DESEMPENHO DOS CANDIDATOS SELECIONADOS PARA COMPOR A EQUIPE DE AVALIAÇÃO (JULGADOR 1 A JULGADOR 6)}

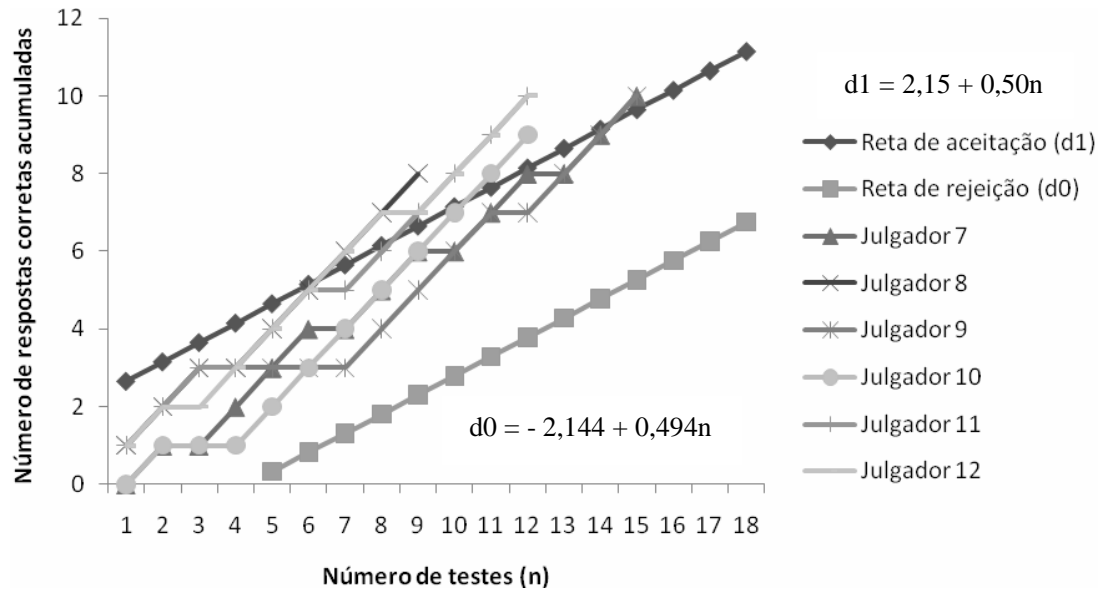

\section{FIGURA 3 - RESULTADO DO DESEMPENHO DOS CANDIDATOS SELECIONADOS PARA COMPOR A EQUIPE DE AVALIAÇÃO (JULGADOR 7 A JULGADOR 12)}

Os doze candidatos selecionados para a avaliação de diferença significativa $(p d \leq 0,001)$ entre extratos hidrossolúveis, produzidos com amêndoas de diferentes classificações, apresentaram acuidade para detectar diferença superior a p0 (0,33). Verificou-se melhor desempenho dos julgadores 4,6 e 8 para os quais foram necessárias apenas nove provas para definir sua inclusão na equipe sensorial. No entanto, os julgadores 1, 2, 5, 7 e 9 necessitaram de testes adicionais aos doze iniciais para atingirem o número suficiente de respostas corretas acumuladas para serem selecionados. Os demais julgadores foram selecionados ao fim dos doze testes. Segundo Bayarri et al. (2008), o julgador ganha experiência quando em testes sucessivos acumula fracassos nos primeiros testes e sucessos nos últimos. Embora diferente, essa situação assemelha-se a que ocorre em perfil sensorial quando julgadores são treinados para reduzir a variância residual. A situação oposta, sucessos acumulados nos primeiros testes e fracassos nos últimos pode sugerir fadiga ou falta de motivação. 
Para 36 respostas no teste triangular, o número mínimo de acertos para indicar diferença significativa a $0,001 \%$ de significância é 22 . Batoques e pedaços apresentaram diferença significativa $(\mathrm{pd} \leq 0,001)$ como matéria-prima para obtenção do extrato hidrossolúvel da amêndoa da castanha de caju, tendo em vista que o número de acertos (23) foi maior que a diferença mínima significativa. $O$ mesmo ocorreu entre bandas e pedaços, com 24 acertos. Somente bandas e batoques não diferiram entre si a $0,001 \%$ de significância como matéria-prima para produção do extrato hidrossolúvel. 0 número de respostas corretas foi de apenas 20 , menor que a diferença mínima significativa. Assim, as amêndoas classificadas como bandas e batoques, do tipo 1, são mais indicadas como matéria-prima para elaboração de extrato hidrossolúvel da ACC.

O resultado obtido apresenta coerência, tendo em vista que bandas (S1) e batoques (B1) revelaram o maior percentual de integridade entre as amêndoas quebradas. A diferença básica entre os dois tipos reside no corte longitudinal (bandas) ou transversal (batoques). As amêndoas quebradas, classificadas como pedaços, apresentam menor percentual de integridade que bandas e batoques em razão de cortes transversais e longitudinais.

Tendo em vista o elevado teor lipídico da amêndoa da castanha de caju, menor percentual de integridade pode estar relacionado a maior nível de oxidação, devido maior área exposta. Além disso, quanto menor o tamanho da amêndoa quebrada maior o tempo de manipulação durante o beneficiamento. As maiores são selecionadas primeiro e seguem para o envase a vácuo ou com injeção de $\mathrm{CO}_{2}$, que evita a oxidação. Por outro lado, as quebradas de menor tamanho ficam mais tempo em contato com o oxigênio atmosférico, principal agente da oxidação lipídica.

As três classes de amêndoas utilizadas (tipo1) para o preparo das amostras são de primeira qualidade e de cor alva ou marfim pálido. Assim, fornecem garantia em termos de sanidade e de coloração similar entre os extratos obtidos com cada classe de amêndoas.

\section{CONCLUSÃO}

A análise sequencial de Wald permitiu selecionar equipe de julgadores para teste triangular com a realização de apenas nove a quinze testes. As amêndoas classificadas como bandas e batoques do tipo 1 não apresentaram diferença sensorial significativa $(\mathrm{pd} \leq 0,001)$, no entanto, diferiram das amêndoas classificadas como pedaços, também do tipo 1. Com isso, bandas e batoques são as amêndoas da castanha de caju mais indicadas como matéria-prima para elaboração de extrato hidrossolúvel.

\section{ABSTRACT \\ SELECTION OF JUDGES AND EVALUATION OF SENSORY DIFFERENCE BETWEEN CASHEW NUT ALMOND HYDROSOLUBLES EXTRACTS (Anacardium occidentale L.)}

The triangle test was applied with 12 selected judges to verify significant difference $(p \leq 0,001)$ in the use of broken cashew nut almonds classified as splits, butts and pieces, all of them type 1 , as raw material to produce an hydrosoluble extract. The samples had been produced triturating cashew nut almonds with different ratios of mineral water. In the selection of judges stage the ratios of almonds:water had been 1:6, $1: 8$ and $1: 10$ and for the discriminative test this ratio was 1:8, having difference enters among samples only in relation to the cashew nut almonds classification. The samples had been packed in polyethylene packages, pasteurized and stored under refrigeration. The selection of judges was carried through triangle test. The results had been evaluated statistically by the sequential analysis of Wald, according to graphical method. The decision was based on the test of hypotheses: $\mathrm{HO}$ : $\mathrm{p} 1 \leq \mathrm{pO} ; \mathrm{H} 1: \mathrm{p} 1>\mathrm{p} 0$, and used the values $\mathrm{p} 0=0.33$, $\mathrm{p} 1=0.66$, for the risks $\alpha=0.05$ and $\beta=0.05$. In the evaluation of significant difference $(p \leq 0.001)$ through the triangle test, the almonds classified as splits and butts of type 1 did not present difference, but differed from 
the almonds classified as pieces of type 1. In view that almonds classified as pieces have a minor percentile of integrity that the others, being thus more susceptible to the lipid oxidation, is indicated the use of splits and butts for the hydrosoluble extract production.

KEY-WORDS: SEQUENTIAL ANALYSIS; TRIANGLE TEST; HYDROSOLUBLE EXTRACT; CASHEW NUT ALMOND.

\section{REFERÊNCIAS}

1 AFI. Association of Food Industries. Departamento de Castanhas \& Produtos Agrícolas, Especificações para amêndoas de castanha de caju. Nova York, abril 1999. Disponível em: <http://www.amberwoodtrading.com/ Specifications/CashewSpecifications.aspx>. Acesso em: 04 ago. 2008.

2 ANDRADE NETO, J. C. Competitividade na pequena produção agroindustrial: estudo na agroindústria da castanha de caju. 2006. 78 p. Dissertação (Mestrado em Engenharia de Produção), Universidade Federal do Rio Grande do Norte. Natal, 2006.

3 ABNT. Associação Brasileira de Normas Técnicas. NBR 12995: teste triangular em análise sensorial de alimentos e bebidas. Rio de Janeiro, 1993

4 BAYARRI, S.; CARBONELL, I.; IZQUIERDO, L.; TÁRREGA, A. Replicated triangle and duo-trio tests: discrimination capacity of assessors evaluated by Bayes' rule. Food Quality and Preference, v. 19, p. 519-523, 2008.

5 BI, JIAN. The double discrimination methods. Food Quality and Preference, v. 12, p. 507-513, 2001.

6 BRASIL. Ministério da Agricultura, Pecuária e Abastecimento. Instrução Normativa nº 62, de 15 de dezembro de 2009. Regulamento técnico da amêndoa da castanha de caju. Diário Oficial [da] República Federativa do Brasil, Brasília, 16 dez. 2009. Seção 1, p. 29.

7 CARBONELL, L.; CARBONELL, I.; IZQUIERDO, L. Triangle tests. Number of discriminators estimated by Bayes' rule. Food Quality and Preference, v. 18, p. 117-120, 2007.

8 CARDARELLI, H. R.; OliVEIRA, A. J. Conservação do leite de castanha-do-pará. Scientia Agricola, v. 57, n. 4, p.617-622, 2000.

9 CHAVES, J. B. P. Cadernos didáticos: análise sensorial: métodos de diferença em avaliação sensorial de alimentos e bebidas. Viçosa: UFV, 2001. 91p.

10 FARIA, E. V.; YOTSUYANAGI, K. Técnicas de análise sensorial. 2. ed. Campinas: ITAL, 2008. 120 p.

11 FELBERG, I.; CABRAL, L.C.; GONÇALVES, E. B.; DELIZA, R. Efeito das condições de extração no rendimento e na qualidade do leite de castanha-do-brasil despeliculada. Boletim do CEPPA, Curitiba, v. 20, n. 1, p. 75-88, jan/jun., 2002.

12 FIGUEIRÊDO JÚNIOR, H. S. Desafios para a cajucultura no Brasil: o comportamento da oferta e da demanda da castanha de caju. Revista Econômica do Nordeste, Fortaleza, v. 37, n.4, p. 550-571, 2006.

13 LAMEIRA, C. P.; COELHO, G. L. V.; MOTHE, C. G. Extração de lipídeos da amêndoa de castanha de caju com $\mathrm{CO}_{2}$ supercrítico. Ciência e Tecnologia de Alimentos, v. 17, n. 4, p. 405-407, 1997.

14 LIMA, A. C.; GARCíA, N. H. P.; LIMA, J. R. Obtenção e caracterização dos principais produtos do caju. Boletim do CEPPA, Curitiba, v. 22, n. 1, p. 133-144, 2004.

15 NASSAR, A. M.; MARINO, M. K.; HERRMANN, I. Desafios para a Coordenação do SAG do caju no Maranhão. In: SEMINÁRIO BRASILEIRO DA NOVA ECONOMIA INSTITUCIONAL, 2001, 2., Campinas. Anais...Campinas: SBNEI, 2004.

16 PARREIRAS, L. E. Negócios solidários em cadeias produtivas: protagonismo coletivo e desenvolvimento sustentável. Rio de Janeiro: IPEA, 2007.

17 PINHEIRO, P. R.; CARVALHO, A. L. A.; CASTRO, A. K. A.; RODRIGUES, M. M.; LIMA, E. M. ZAPROS LM aplicado ao processo de industrialização da castanha de caju. 2006. Disponível em: <http://www.abepro.org.br/biblioteca/ ENEGEP2006_TR450301_7981.pdf>. Acesso em: 23 abr. 2008.

18 SOUZA FILHO, M. S. M.; MAIA, G. A.; HOLANDA, L. F. F.; ORIÁ, H. F.; OLIVEIRA, G. S. F.; FIGUEIREDO, R. W. Composição percentual dos ácidos graxos da fração lipídica da amêndoa da castanha de caju de diferentes clones de caju (Anacardium occidentale L.). Ciência Agronômica, Fortaleza, v. 22, n.1/2, p. 43-46, jun./dez. 1991. 
19 STONE, H.; SIDEL, J. Sensory evaluation practices. $2^{\text {nd }}$ ed. London: Academic Press, 1993. 338 p.

20 USAID. United States Agency International Development. Análise da indústria de castanha de caju: inserção de micro e pequenas empresas no mercado internacional. Washington, 2006. 77 p.

21 USDA. United States Department of Agriculture. National database for Standard Reference, 2008. Disponível em: <http://www.usda.ogr>. Acesso em: 20 abr. 2008. 